\title{
A fixed point theorem for generalized contractions involving $w$-distances on complete quasi-metric spaces
}

\author{
Carmen Alegre, Josefa Marín and Salvador Romaguera* \\ Dedicated to Professor W. Takahashi on the occasion of his 70th birthday
}

\author{
"Correspondence: \\ sromague@mat.upv.es \\ Instituto Universitario de \\ Matemática Pura y Aplicada, \\ Universitat Politècnica de València, \\ Valencia, 46022, Spain
}

\begin{abstract}
We obtain a fixed point theorem for generalized contractions on complete quasi-metric spaces, which involves $w$-distances and functions of Meir-Keeler and Jachymski type. Our result generalizes in various directions the celebrated fixed point theorems of Boyd and Wong, and Matkowski. Some illustrative examples are also given.

MSC: 47H10;54H25; 54E50
\end{abstract}

Keywords: fixed point; generalized contraction; $w$-distance; complete quasi-metric space

\section{Introduction and preliminaries}

In their celebrated paper [1], Kada, Suzuki and Takahashi introduced and studied the notion of a $w$-distance on a metric space. By using that notion they obtained, among other results, generalizations of the nonconvex minimization theorem of Takahashi [2], of Caristi's fixed point theorem [3] and of Ekeland's variational principle [4], as well as a general fixed point theorem that improves fixed point theorems of Subrahmanyam [5], Kannan [6] and Cirić [7]. This study was continued by Suzuki and Takahashi [8], and by Park [9] who extended several results from [1] to quasi-metric spaces. Park's approach was successful continued by Al-Homidan, Ansari and Yao [10], who obtained, among other interesting results, quasi-metric versions of Caristi-Kirk's fixed point theorem and Nadler's fixed point theorem by using $Q$-functions (a slight generalization of $w$-distances). More recently, Latif and Al-Mezel [11], and Marín et al. [12-14] have proved some fixed point theorems both for single-valued and multi-valued mappings in complete quasi-metric spaces and preordered quasi-metric spaces by using $Q$-functions and $w$-distances, and generalizing in this way well-known fixed point theorems of Mizoguchi and Takahashi [15], Bianchini and Grandolfi [16], and Boyd and Wong [17], respectively.

In this paper we shall obtain a fixed point theorem for generalized contractions with respect to $w$-distances on complete quasi-metric spaces from which we deduce $w$-distance versions of Boyd and Wong's fixed point theorem [17] and of Matkowski's fixed point theorem [18]. Our approach uses a kind of functions considered by Jachymski in [19, Corollary of Theorem 2] and that generalizes the notion of a function of Meir-Keeler type.

○2014 Alegre et al.; licensee Springer. This is an Open Access article distributed under the terms of the Creative Commons Attribution License (http://creativecommons.org/licenses/by/2.0), which permits unrestricted use, distribution, and reproduction in any medium, provided the original work is properly cited. 
In the sequel the letters $\mathbb{R}^{+}, \mathbb{N}$ and $\omega$ will denote the set of non-negative real numbers, the set of positive integer numbers and the set of non-negative integer numbers, respectively.

By a quasi-metric on a set $X$ we mean a function $d: X \times X \rightarrow \mathbb{R}^{+}$such that for all $x, y, z \in X$ :

(i) $d(x, y)=d(y, x)=0 \Leftrightarrow x=y$, and

(ii) $d(x, y) \leq d(x, z)+d(z, y)$.

A quasi-metric space is a pair $(X, d)$ such that $X$ is a set and $d$ is a quasi-metric on $X$.

Each quasi-metric $d$ on a set $X$ induces a topology $\tau_{d}$ on $X$ which has as a base the family of open balls $\left\{B_{d}(x, r): x \in X, \varepsilon>0\right\}$, where $B_{d}(x, \varepsilon)=\{y \in X: d(x, y)<\varepsilon\}$ for all $x \in X$ and $\varepsilon>0$.

Given a quasi-metric $d$ on $X$, the function $d^{-1}$ defined by $d^{-1}(x, y)=d(y, x)$ for all $x, y \in X$, is also a quasi-metric on $X$, and the function $d^{s}$ defined by $d^{s}(x, y)=\max \{d(x, y), d(y, x)\}$ for all $x, y \in X$, is a metric on $X$.

There exist several different notions of Cauchy sequence and of complete quasi-metric space in the literature (see e.g. [20]). In this paper we shall use the following general notion.

A quasi-metric space $(X, d)$ is called complete if every Cauchy sequence $\left(x_{n}\right)_{n \in \omega}$ in the metric space $\left(X, d^{s}\right)$ converges with respect to the topology $\tau_{d^{-1}}$ (i.e., there exists $z \in X$ such that $\left.d\left(x_{n}, z\right) \rightarrow 0\right)$.

Definition $1([9,10])$ A $w$-distance on a quasi-metric space $(X, d)$ is a function $q: X \times X \rightarrow$ $\mathbb{R}^{+}$satisfying the following three conditions:

(W1) $q(x, y) \leq q(x, z)+q(z, y)$ for all $x, y, z \in X$;

(W2) $q(x, \cdot): X \rightarrow \mathbb{R}^{+}$is lower semicontinuous on $\left(X, \tau_{d^{-1}}\right)$ for all $x \in X$;

(W3) for each $\varepsilon>0$ there exists $\delta>0$ such that $q(x, y) \leq \delta$ and $q(x, z) \leq \delta$ imply $d(y, z) \leq \varepsilon$

Several examples of $w$-distances on quasi-metric spaces may be found in [9-12].

Note that if $d$ is a metric on $X$ then it is a $w$-distance on $(X, d)$. Unfortunately, this does not hold for quasi-metric spaces, in general. Indeed, in [12, Lemma 2.2] there was observed the following.

Lemma 1 If $q$ is a $w$-distance on a quasi-metric space $(X, d)$, then for each $\varepsilon>0$ there exists $\delta>0$ such that $q(x, y) \leq \delta$ and $q(x, z) \leq \delta$ imply $d^{s}(y, z) \leq \varepsilon$.

It follows from Lemma 1 (see [12, Proposition 2.3]) that if a quasi-metric $d$ on $X$ is also a $w$-distance on $(X, d)$, then the topologies induced by $d$ and by the metric $d^{s}$ coincide, so $\left(X, \tau_{d}\right)$ is a metrizable topological space.

\section{Results and examples}

Meir and Keeler proved in [21] that if $f$ is a self-map of a complete metric space $(X, d)$ satisfying the condition that for each $\varepsilon>0$ there is $\delta>0$ such that, for any $x, y \in X$, with $\varepsilon \leq d(x, y)<\varepsilon+\delta$ we have $d(f x, f y)<\varepsilon$, then $f$ has a unique fixed point $z \in X$ and $f^{n} x \rightarrow z$ for all $x \in X$.

This well-known result suggests the notion of a Meir-Keeler function:

A function $\phi: \mathbb{R}^{+} \rightarrow \mathbb{R}^{+}$is said to be a Meir-Keeler function if $\phi(0)=0$, and satisfies the following condition: 
(MK) For each $\varepsilon>0$ there exists $\delta>0$ such that

$$
\varepsilon \leq t<\varepsilon+\delta \quad \text { implies } \quad \phi(t)<\varepsilon, \quad \text { for all } t \in \mathbb{R}^{+} .
$$

Remark 1 It is obvious that if $\phi$ is a Meir-Keeler function then $\phi(t)<t$ for all $t>0$.

Later on, Jachymski proved in [19] the following interesting result and showed that both Boyd and Wong's fixed point theorem and Matkowski's fixed point theorem are easy consequences of it.

Theorem 1 ([19, Corollary of Theorem 2]) Let $f$ be a self-map of a complete metric space $(X, d)$ such that $d(f x, f y)<d(x, y)$ for $x \neq y$, and $d(f x, f y) \leq \phi(d(x, y))$ for all $x, y \in X$, where $\phi: \mathbb{R}^{+} \rightarrow \mathbb{R}^{+}$satisfies the condition

(Ja) for each $\varepsilon>0$ there exists $\delta>0$ such that for any $t \in \mathbb{R}^{+}$,

$$
\varepsilon<t<\varepsilon+\delta \quad \text { implies } \quad \phi(t) \leq \varepsilon
$$

Then $f$ has a unique fixed point $z \in X$ and $f^{n} x \rightarrow z$ for all $x \in X$.

Theorem 1 suggests the following notion:

A function $\phi: \mathbb{R}^{+} \rightarrow \mathbb{R}^{+}$is said to be a Jachymski function if $\phi(0)=0$ and it satisfies condition (Ja) of Theorem 1 .

Remark 2 Obviously, each Meir-Keeler function is a Jachymski function. However, the converse does not follow even in the case that $\phi(t)<t$ for all $t>0$ : Indeed, let $\phi: \mathbb{R}^{+} \rightarrow \mathbb{R}^{+}$ defined as $\phi(t)=0$ for all $t \in[0,1]$ and $\phi(t)=1$ otherwise. Clearly $\phi$ is a Jachymski function such that $\phi(t)<t$ for all $t>0$. Finally, for $\varepsilon=1$ and any $\delta>0$ we have $\phi(\varepsilon+\delta / 2)=\varepsilon$, so $\phi$ is not a Meir-Keeler function.

Now we establish the main result of this paper.

Theorem 2 Let $f$ be a self-map of a complete quasi-metric space $(X, d)$. If there exist a $w$-distance $q$ on $(X, d)$ and a Jachymski function $\phi: \mathbb{R}^{+} \rightarrow \mathbb{R}^{+}$such that $\phi(t)<t$ for all $t>0$, and

$$
q(f x, f y) \leq \phi(q(x, y))
$$

for all $x, y \in X$, then $f$ has a unique fixed point $z \in X$. Moreover $q(z, z)=0$.

Proof Fix $x_{0} \in X$. For each $n \in \omega$ let $x_{n}=f^{n} x_{0}$. Then

$$
q\left(x_{n+1}, x_{n+2}\right) \leq \phi\left(q\left(x_{n}, x_{n+1}\right)\right),
$$

for all $n \in \omega$.

First, we shall prove that $\left\{x_{n}\right\}_{n \in \omega}$ is a Cauchy sequence in $\left(X, d^{s}\right)$.

To this end put $r_{n}=q\left(x_{n}, x_{n+1}\right)$ for all $n \in \omega$. 
If there is $n_{0} \in \omega$ such that $r_{n_{0}}=0$, then $r_{n}=0$ for all $n \geq n_{0}$ by (2) and our assumption that $\phi(0)=0$. Therefore $q\left(x_{n}, x_{m}\right)=0$ whenever $m>n \geq n_{0}$ by condition (W1), and consequently, $d^{s}\left(x_{n}, x_{m}\right)=0$ by Lemma 1 . Thus $x_{n}=x_{n_{0}+1}$ for all $n \geq n_{0}+1$.

Otherwise, we assume, without loss of generality, that $r_{n+1}<r_{n}$ for all $n \in \omega$. Then $\left\{r_{n}\right\}_{n \in \omega}$ converges to some $r \in \mathbb{R}^{+}$. Of course, $r<r_{n}$ for all $n \in \omega$.

If $r>0$ there exists $\delta=\delta(r)$ such that

$$
r<t<r+\delta \Rightarrow \phi(t) \leq r .
$$

Take $n_{\delta} \in \mathbb{N}$ such that $r_{n}<r+\delta$ for all $n \geq n_{\delta}$. Therefore $\phi\left(r_{n}\right) \leq r$, so by condition (2), $r_{n+1} \leq r$ for all $n \geq n_{\delta}$, a contradiction. Consequently $r=0$.

Now choose an arbitrary $\varepsilon>0$. There exists $\delta=\delta(\varepsilon)$, with $\delta \in(0, \varepsilon)$, for which conditions (W3) and (Ja) hold. Similarly, for $\delta / 2$ there exists $\mu=\mu(\delta / 2)$, with $\mu \in(0, \delta / 2)$ for which conditions (W3) and (Ja) also hold, i.e.,

$q(x, y) \leq \mu$ and $q(x, z) \leq \mu$, imply $d(y, z) \leq \delta / 2$, and for any $t>0, \delta / 2<t<\delta / 2+\mu$

implies $\phi(t) \leq \delta / 2$.

Since $r_{n} \rightarrow 0$, there exists $k_{0} \in \mathbb{N}$ such that $r_{n}<\mu$ for all $n \geq k_{0}$.

By using a similar technique to the one given by Jachymski in [19, Theorem 2] we shall prove, by induction, that for each $k \geq k_{0}$ and each $n \in \mathbb{N}$, we have

$$
q\left(x_{k}, x_{n+k}\right)<\frac{\delta}{2}+\mu .
$$

Indeed, fix $k \geq k_{0}$. Since $q\left(x_{k}, x_{k+1}\right)<\mu$, condition (3) follows for $n=1$.

Assume that (3) holds for some $n \in \mathbb{N}$. We shall distinguish two cases.

- Case 1: $q\left(x_{k}, x_{n+k}\right)>\delta / 2$. Then we deduce from the induction hypothesis and condition (Ja) that

$$
\phi\left(q\left(x_{k}, x_{n+k}\right)\right) \leq \delta / 2,
$$

so by (1), $q\left(x_{k+1}, x_{n+k+1}\right) \leq \delta / 2$. Therefore

$$
q\left(x_{k}, x_{n+k+1}\right) \leq q\left(x_{k}, x_{k+1}\right)+q\left(x_{k+1}, x_{n+k+1}\right)<\mu+\frac{\delta}{2} .
$$

- Case 2: $q\left(x_{k}, x_{n+k}\right) \leq \delta / 2$.

If $q\left(x_{k}, x_{n+k}\right)=0$, we deduce that $q\left(x_{k+1}, x_{n+k+1}\right)=0$ by (1). So, by (W1),

$$
q\left(x_{k}, x_{n+k+1}\right) \leq q\left(x_{k}, x_{k+1}\right)<\mu<\mu+\frac{\delta}{2} .
$$

If $q\left(x_{k}, x_{n+k}\right)>0$, we deduce that $\phi\left(q\left(x_{k}, x_{n+k}\right)\right)<q\left(x_{k}, x_{n+k}\right) \leq \delta / 2$, so

$$
\begin{aligned}
q\left(x_{k}, x_{n+k+1}\right) & \leq q\left(x_{k}, x_{k+1}\right)+q\left(x_{k+1}, x_{n+k+1}\right) \\
& \leq q\left(x_{k}, x_{k+1}\right)+\phi\left(q\left(x_{k}, x_{n+k}\right)\right)<\mu+\frac{\delta}{2} .
\end{aligned}
$$

Now take $i, j \in \mathbb{N}$ with $i, j>k$. Then $i=n+k$ and $j=m+k$ for some $n, m \in \mathbb{N}$. Hence, by (3),

$$
q\left(x_{k}, x_{i}\right)=q\left(x_{k}, x_{n+k}\right)<\frac{\delta}{2}+\mu<\delta \quad \text { and } \quad q\left(x_{k}, x_{j}\right)=q\left(x_{k}, x_{m+k}\right)<\frac{\delta}{2}+\mu<\delta .
$$


Now, from Lemma 1 it follows that $d^{s}\left(x_{i}, x_{j}\right) \leq \varepsilon$ whenever $i, j>k$. We conclude that $\left\{x_{n}\right\}_{n \in \mathbb{N}}$ is a Cauchy sequence in $\left(X, d^{s}\right)$.

Since $(X, d)$ is complete, there exists $z \in X$ such that $d\left(x_{n}, z\right) \rightarrow 0$.

Next we show that $q\left(x_{n}, z\right) \rightarrow 0$ : Indeed, choose an arbitrary $\varepsilon>0$. We have proved (see (3)) that there is $k_{0} \in \mathbb{N}$ such that $q\left(x_{k}, x_{n+k}\right)<\varepsilon$ for all $k \geq k_{0}$ and $n \in \mathbb{N}$. Fix $k \geq k_{0}$. Since $d\left(x_{n}, z\right) \rightarrow 0$ it follows from condition (W2) that, for $n$ sufficiently large,

$$
q\left(x_{k}, z\right)<q\left(x_{k}, x_{n+k}\right)+\varepsilon .
$$

Hence $q\left(x_{k}, z\right)<2 \varepsilon$ for all $k \geq k_{0}$. We deduce that $q\left(x_{n}, z\right) \rightarrow 0$.

From (1) it follows that $q\left(x_{n+1}, f z\right) \rightarrow 0$. So $d^{s}(z, f z)=0$ by Lemma 1 . Consequently $z=f z$, i.e., is a fixed point of $f$. Furthermore $q(z, z)=0$. In fact, otherwise we have

$$
q(z, z)=q(f z, f z) \leq \phi(q(z, z))<q(z, z)
$$

a contradiction.

Finally, let $u \in X$ such that $u=f u$ and $u \neq z$. If $q(u, z)>0$ we deduce that

$$
q(u, z)=q(f u, f z) \leq \phi(q(u, z))<q(u, z),
$$

a contradiction. So $q(u, z)=0$. Similarly we check that $q(u, u)=0$. Since $q(z, z)=0$, we deduce from Lemma 1 that $d^{s}(u, z)=0$, i.e., $u=z$. We conclude that $z$ is the unique fixed point of $f$.

Corollary 1 Let $f$ be a self-map of a complete metric space $(X, d)$. If there exist a $w$-distance $q$ on $(X, d)$ and a Jachymski function $\phi: \mathbb{R}^{+} \rightarrow \mathbb{R}^{+}$such that $\phi(t)<t$ for all $t>0$, and

$$
q(f x, f y) \leq \phi(q(x, y))
$$

for all $x, y \in X$, then $f$ has a unique fixed point $z \in X$. Moreover $q(z, z)=0$.

Corollary 2 Let $f$ be a self-map of a complete quasi-metric space $(X, d)$. If there exist a $w$-distance $q$ on $(X, d)$ and a Meir-Keeler function $\phi: \mathbb{R}^{+} \rightarrow \mathbb{R}^{+}$such that

$$
q(f x, f y) \leq \phi(q(x, y))
$$

for all $x, y \in X$, then $f$ has a unique fixed point $z \in X$. Moreover $q(z, z)=0$.

Proof Apply Remarks 1 and 2, and Theorem 2.

Corollary 3 [13] Let $f$ be a self-map of a complete quasi-metric space $(X, d)$. If there exist a w-distance $q$ on $(X, d)$ and a right upper semicontinuous function $\phi: \mathbb{R}^{+} \rightarrow \mathbb{R}^{+}$such that $\phi(0)=0, \phi(t)<t$ for all $t>0$, and

$$
q(f x, f y) \leq \phi(q(x, y))
$$

for all $x, y \in X$, then $f$ has a unique fixed point $z \in X$. Moreover $q(z, z)=0$. 
Proof It suffices to show that $\phi$ is a Meir-Keeler function. Assume the contrary. Then there exist $\varepsilon>0$ and a sequence $\left\{t_{n}\right\}_{n \in \mathbb{N}}$ of positive real numbers such that $\varepsilon \leq t_{n}<\varepsilon+1 / n$ but $\phi\left(t_{n}\right) \geq \varepsilon$ for all $n \in \mathbb{N}$. Since $\varepsilon-\phi(\varepsilon)>0$, it follows from right upper semicontinuity of $\phi$ that $\phi\left(t_{n}\right)-\phi(\varepsilon)<\varepsilon-\phi(\varepsilon)$ eventually, i.e., $\phi\left(t_{n}\right)<\varepsilon$, a contradiction. We conclude that $f$ has a unique fixed point by Corollary 2 .

Corollary 4 Let $f$ be a self-map of a complete quasi-metric space $(X, d)$. If there exist a $w$-distance $q$ on $(X, d)$ and a non-decreasing function $\phi: \mathbb{R}^{+} \rightarrow \mathbb{R}^{+}$such that $\phi(0)=0$, $\phi^{n}(t) \rightarrow 0$ for all $t>0$, and

$$
q(f x, f y) \leq \phi(q(x, y))
$$

for all $x, y \in X$, then $f$ has a unique fixed point $z \in X$. Moreover $q(z, z)=0$.

Proof Again it suffices to show that $\phi$ is a Meir-Keeler function. Assume the contrary. Then there exist $\varepsilon>0$ and a sequence $\left\{t_{n}\right\}_{n \in \mathbb{N}}$ of positive real numbers such that $\varepsilon \leq t_{n}<$ $\varepsilon+1 / n$ but $\phi\left(t_{n}\right) \geq \varepsilon$ for all $n \in \mathbb{N}$. Since $\phi$ is non-decreasing we deduce that $\phi(t) \geq \varepsilon$ whenever $t \geq \varepsilon$. Hence $\phi^{n}(t) \geq \varepsilon$ whenever $t \geq \varepsilon$, which contradicts the hypothesis that $\phi^{n}(t) \rightarrow 0$ for all $t>0$. We conclude that $f$ has a unique fixed point by Corollary 2 .

Remark 3 In [22] the authors proved Corollary 2 for the case that $(X, d)$ is a complete metric space. Note also that Boyd and Wong's fixed point theorem [17] and Matkowski's fixed point theorem [18] are special cases of Corollaries 3 and 4, respectively, when $(X, d)$ is a complete metric space and $q$ is the metric $d$.

We conclude the paper with some examples that illustrate and validate the obtained results.

The first example shows that condition ' $\phi(t)<t$ for all $t>0$ ' in Theorem 2 cannot be omitted.

Example 1 Let $X=\{0,1\}$ and let $d$ be the discrete metric on $X$, i.e., $d(x, x)=0$ for all $x \in X$ and $d(x, y)=1$ whenever $x \neq y$. Let $f: X \rightarrow X$ defined as $f 0=1$ and $f 1=0$, and $\phi: \mathbb{R}^{+} \rightarrow \mathbb{R}^{+}$ defined as $\phi(1)=1$ and $\phi(t)=0$ for all $x \in \mathbb{R}^{+} \backslash\{1\}$. It is clear that $\phi$ is a Jachysmki function such that

$$
d(f x, f y) \leq \phi(d(x, y))
$$

for all $x, y \in X$. However, $f$ has no fixed point.

The next is an example where we can apply Theorem 2 for an appropriate $w$-distance $q$ on a complete quasi-metric space $(X, d)$ but not for $d$. Moreover, Corollary 1 cannot be applied for any $w$-distance on the metric space $\left(X, d^{s}\right)$.

Example 2 Let $X=\omega$ and let $d$ be the quasi-metric on $X$ defined as

$$
\begin{aligned}
& d(x, x)=0 \quad \text { for all } x \in X \\
& d(n, 0)=1 / n \quad \text { for all } n \in \mathbb{N} ;
\end{aligned}
$$




$$
\begin{aligned}
& d(0, n)=1 \quad \text { for all } n \in \mathbb{N} ; \\
& d(n, m)=|1 / n-1 / m| \text { for all } n, m \in \mathbb{N} .
\end{aligned}
$$

Clearly $(X, d)$ is complete (observe that $\{n\}_{n \in \mathbb{N}}$ is a Cauchy sequence in $\left(X, d^{s}\right)$ with $d(n, 0) \rightarrow 0)$.

Let $q$ be the $w$-distance on $(X, d)$ given by $q(x, y)=y$ for all $x, y \in X$.

Now define $f: X \rightarrow X$ as $f 0=0$ and $f n=n-1$ for all $n \in \mathbb{N}$, and $\phi: \mathbb{R}^{+} \rightarrow \mathbb{R}^{+}$as $\phi(0)=0$ and $\phi(t)=n-1$ where $t \in(n-1, n], n \in \mathbb{N}$.

It is routine to check that $\phi$ is a Jachymski function satisfying $\phi(t)<t$ for all $t>0$ (in fact, it is a Meir-Keeler function).

Since $q(f x, f 0)=0$ for all $x \in X$, and for each $n, m \in X$ with $m \neq 0$, we have

$$
q(f n, f m)=f m=m-1=\phi(m)=\phi(q(n, m))
$$

it follows that all conditions of Theorem 2 are satisfied. In fact $z=0$ is the unique fixed point of $f$.

However, the contraction condition (1) is not satisfied for $d$. Indeed, for any $n>1$ we have

$$
d(f 0, f n)=d(0, n-1)=1>0=\phi(1)=\phi(d(0, n)) .
$$

Finally, note that we cannot apply Corollary 1 because $\left(X, d^{s}\right)$ is not complete (observe that $\{n\}_{n \in \mathbb{N}}$ is a Cauchy sequence in $\left(X, d^{s}\right)$ that does not converge in $\left.\left(X, d^{s}\right)\right)$.

We conclude with an example where we can apply Corollary 2 but not Corollaries 3 and 4 .

Example 3 Let $d$ be the quasi-metric on $\mathbb{R}^{+}$given by $d(x, y)=\max \{y-x, 0\}$ for all $x, y \in \mathbb{R}^{+}$. Since $d^{s}$ is the usual metric on $\mathbb{R}^{+}$it immediately follows that $\left(\mathbb{R}^{+}, d\right)$ is complete.

Define $q: \mathbb{R}^{+} \times \mathbb{R}^{+} \rightarrow \mathbb{R}^{+}$as $q(x, y)=y$. It is clear that $q$ is a $w$-distance on $\left(\mathbb{R}^{+}, d\right)$.

Now let $\phi: \mathbb{R}^{+} \rightarrow \mathbb{R}^{+}$, defined by $\phi(t)=t / 2$ if $t \in(1,2]$, and $\phi(t)=0$ otherwise.

Then $\phi$ is a Meir-Keeler function: Indeed, we first note that $\phi(0)=0$. Now, given $\varepsilon>0$ we distinguish the following cases:

(1) if $0<\varepsilon<1$, we take $\delta=1-\varepsilon$, and thus, from $\varepsilon \leq t<\varepsilon+\delta=1$, it follows $\phi(t)=0<\varepsilon$;

(2) if $\varepsilon=1$, we take $\delta=1 / 2$, and thus, from $1<t<3 / 2$, it follows $\phi(t)=t / 2<3 / 4<\varepsilon$, whereas $\phi(1)=0<\varepsilon$;

(3) if $1<\varepsilon<2$, we take $\delta=2-\varepsilon$, and thus, from $\varepsilon \leq t<\varepsilon+\delta=2$, it follows $\phi(t)=t / 2<1<\varepsilon ;$

(4) if $\varepsilon \geq 2$, we fix $\delta>0$, and thus, from $\varepsilon \leq t<\varepsilon+\delta$, it follows $\phi(t)<\varepsilon$ because $\phi(2)=1$ and $\phi(t)=0$ for $t>2$.

Finally, taking $f=\phi$, we obtain $q(f x, f y) \leq \phi(q(x, y))$ for all $x, y \in X$, because

$$
q(f x, f y)=f y=\phi(y)=\phi(q(x, y))
$$

Therefore, all conditions of Corollary 2 are satisfied. In fact, $z=0$ is the unique fixed point of $f$. 
However, $\phi$ is not right upper semicontinuous at $t=1$, so we cannot apply Corollary 3 . Similarly, we cannot apply Corollary 4 because $\phi$ is not a non-decreasing function.

Observe also that the $w$-distance $q$ cannot be replaced by the quasi-metric $d$ because for $1<y \leq 2$ we have

$$
d(f 1, f y)=d\left(0, \frac{y}{2}\right)=\frac{y}{2}>0=\phi(y-1)=\phi(d(1, y)) .
$$

\section{Competing interests}

The authors declare that they have no competing interests.

\section{Authors' contributions}

The three authors contributed equally in writing this article. They read and approved the final manuscript.

\section{Acknowledgements}

The authors are grateful to the referees for several useful suggestions. They also thank the support of the Ministry of Economy and Competitiveness of Spain, Grant MTM2012-37894-C02-01.

Received: 30 September 2013 Accepted: 4 February 2014 Published: 14 Feb 2014

\section{References}

1. Kada, O, Suzuki, T, Takahashi, W: Nonconvex minimization theorems and fixed point theorems in complete metric spaces. Math. Jpn. 44, 381-391 (1996)

2. Takahashi, W: Existence theorems generalizing fixed point theorems for multivalued mappings. In: Théra, MA, Baillon, JB (eds.) Fixed Point Theory and Applications. Pitman Research Notes in Mathematics Series, vol. 252, pp. 397-406. Longman, Harlow (1991)

3. Caristi, J: Fixed point theorems for mappings satisfying inwardness conditions. Trans. Am. Math. Soc. 215, 241-251 (1976)

4. Ekeland, I: Nonconvex minimization problems. Bull. Am. Math. Soc. 1, 443-474 (1979)

5. Subrahmanyam, PV: Remarks on some fixed point theorems related to Banach's contraction principle. J. Math. Phys. Sci. 8, 445-457 (1974). Erratum 9, 195 (1975)

6. Kannan, R: Some results on fixed points. II. Am. Math. Mon. 76, 405-408 (1969)

7. Ćirić, L: A generalization of Banach's contraction principle. Proc. Am. Math. Soc. 45, 267-273 (1974)

8. Suzuki, T, Takahashi, W: Fixed point theorems and characterizations of metric completeness. Topol. Methods Nonlinear Anal. 8, 371-382 (1996)

9. Park, S: On generalizations of the Ekeland-type variational principles. Nonlinear Anal. TMA 39, 881-889 (2000)

10. Al-Homidan, S, Ansari, QH, Yao, JC: Some generalizations of Ekeland-type variational principle with applications to equilibrium problems and fixed point theory. Nonlinear Anal. TMA 69, 126-139 (2008)

11. Latif, A, Al-Mezel, SA: Fixed point results in quasimetric spaces. Fixed Point Theory Appl. 2011, Article ID 178306 (2011)

12. Marín, J, Romaguera, S, Tirado, P: Q-functions on quasi-metric spaces and fixed points for multivalued maps. Fixed Point Theory Appl. 2011, Article ID 603861 (2011)

13. Marín, J, Romaguera, S, Tirado, P: Weakly contractive multivalued maps and $w$-distances on complete quasi-metric spaces. Fixed Point Theory Appl. 2011, Article ID 2 (2011)

14. Marín, J, Romaguera, S, Tirado, P: Generalized contractive set-valued maps on complete preordered quasi-metric spaces. J. Funct. Spaces Appl. 2013, Article ID 269246 (2013)

15. Mizoguchi, N, Takahashi, W: Fixed point theorems for multivalued mappings on complete metric spaces. J. Math Anal. Appl. 141, 177-188 (1989)

16. Bianchini, RM, Grandolfi, M: Trasformazioni di tipo contrattivo generalizzato in uno spazio metrico. Atti Accad. Naz. Lincei, Rend. Cl. Sci. Fis. Mat. Nat. (8) 45, 212-216 (1968)

17. Boyd, DW, Wong, JSW: On nonlinear contractions. Proc. Am. Math. Soc. 20, 458-464 (1969)

18. Matkowski, J: Integrable solutions of functional equations. Diss. Math. 127, 1-68 (1975)

19. Jachymski, J: Equivalent conditions and the Meir-Keeler type theorems. J. Math. Anal. Appl. 194, $293-303$ (1995)

20. Künzi, HPA: Nonsymmetric distances and their associated topologies: about the origins of basic ideas in the area of asymmetric topology. In: Aull, CE, Lowen, R (eds.) Handbook of the History of General Topology, vol. 3, pp. $853-968$. Kluwer Academic, Dordrecht (2001)

21. Meir, A, Keeler, E: A theorem on contraction mappings. J. Math. Anal. Appl. 28, 326-329 (1969)

22. Alegre, C, Marín, J, Romaguera, S: Fixed points for generalized contractions with respect to $W$-distances and Meir-Keeler functions. In: Proceedings of the Conference in Applied Topology WiAT'13, Bilbao, Spain, pp. 53-58 (2013)

10.1186/1687-1812-2014-40

Cite this article as: Alegre et al.: A fixed point theorem for generalized contractions involving $w$-distances on complete quasi-metric spaces. Fixed Point Theory and Applications 2014, 2014:40 